Educación Física y Ciencia, vol. 21, nº 1, e073, enero-marzo 2019. ISSN 2314-2561

Universidad Nacional de La Plata.

Facultad de Humanidades y Ciencias de la Educación.

Departamento de Educación Física

\title{
Caracterização morfofuncional de praticantes do brazilian jiu-jitsu
}

Morphfunctional characterization of Brazilian jiu-jitsu practice

\author{
Rafaela Silva Bricio Rezende \\ Centro de Ciências Aplicadas à Educação e Saúde (CeCAES) - \\ IFSULDEMINAS, Campus Muzambinho, Brasil \\ rafaelaeducacaofisica@gmail.com
}

Marcio Luiz de Almeida Bueno

IFSULDEMINAS, Campus Muzambinho, Brasil

marcio.bueno@muz.ifsuldeminas.edu.br

Wonder Passoni Higino

Centro de Ciências Aplicadas à Educação e Saúde (CeCAES) -

IFSULDEMINAS, Campus Muzambinho, Brasil

wonderhigino@gmail.com

\begin{abstract}
Resumo:
O objetivo deste estudo foi caracterizar o perfil morfofuncional de atletas amadores da modalidade Brazilian Jiu-jitsu (BJJ), fazendo uma comparação por sexo. Participaram, 19 praticantes da modalidade (7 mulheres: 21,29 \pm 4,68 anos de idade; 25,84 $\pm 3,90 \mathrm{~kg} /$ $\mathrm{m} 2$ de IMC; $22,03 \pm 8,20 \%$ de gordura corporal e 12 homens: 22,03 \pm 6,04 anos de idade; 23,98 \pm 4,08 kg/m2 de IMC; 14,53 $\pm 8,66 \%$ de gordura corporal) a pelo menos um ano e com nível de graduação entre faixa branca e azul. Estes foram submetidos a avaliações de composição corporal, flexibilidade, força de preensão manual e potência de membros inferiores. Após as avaliações a comparação entre os sexos foi realizada através do teste $t$ Student para amostrar independentes $(\mathrm{p} \leq 0,05)$. Diante da análise as únicas variáveis que apresentaram diferenças significantes foram aquelas relacionadas a capacidade de força de preensão manual (direita: $47,35 \pm 8,77 \mathrm{~N}$ e $30,28 \pm 6,65 \mathrm{~N}$; esquerda: $40,75 \pm 8,45 \mathrm{~N}$ e $25,57 \pm 5,94 \mathrm{~N})$ e altura do salto vertical $(33,34 \pm 4,98 \mathrm{~cm}$ e $23,33 \pm 4,30 \mathrm{~cm})$, sendo estatisticamente superiores para os homens. As demais variáveis não apresentaram diferenças significantes. Conclui-se que os homens apresentam maiores níveis de força em comparação as mulheres.
\end{abstract}

Palavras-CHAVE: Brazilian Jiu-jitsu, Caracterização morfofuncional, Sexo.

\begin{abstract}
:
The objective of this study was to characterize the morphofunctional profile of Brazilian Jiu-jitsu (BJJ) amateur athletes, making a comparison by gender. 19 practitioners of the modality participated in the study (7 women: $21.29 \pm 4.68$ years of age, $25.84 \pm 3.90$ $\mathrm{kg} / \mathrm{m} 2$ BMI, $22.03 \pm 8.20 \%$ body fat and 12 men: $22,03 \pm 6.04$ years of age, $23.98 \pm 4.08 \mathrm{~kg} / \mathrm{m} 2$ of BMI, $14.53 \pm 8.66 \%$ of body fat) for at least one year and with graduation level from white belt to blue. These were submitted to assessments related to body composition and flexibility, manual grip strength and lower limb power. After the evaluations, a comparison between genders was performed through the Student $\mathrm{t}$ test to sample independent $(\mathrm{p} \leq 0.05)$. The only variables that presented significant differences were those related to manual grip strength (right $-47.35 \pm 8.77 \mathrm{~N}$ and $30.28 \pm 6.65 \mathrm{~N}$, left $-40.75 \pm 8.45 \mathrm{~N}$ and $25.57 \pm 5,94 \mathrm{~N}$ ) and height of the vertical jump $(33.34 \pm 4.98 \mathrm{~cm}$ and $23.33 \pm 4.30 \mathrm{~cm})$, being statistically superior for men. The other variables did not present significant differences. We concluded that men have higher levels of strength when compared to women.
\end{abstract}

KEYWORDS: Brazilian Jiu-jitsu, Characterize the morphofunctional, Gender. 


\section{INTRODUÇÃO}

O Jiu-jitsu, que traduzido significa arte suave, foi criado e praticado por monges budistas na Índia em 500 a.C que, preocupados com a defesa pessoal, desenvolveram técnicas baseadas nos princípios do equilíbrio dosistema de articulação do corpo e das alavancas, evitando o uso de armas e força. Após originar-se na Índia, acredita-se que o Jiu- jitsu tenha caminhado primeiramente para a China e, dela, para o Japão no século II d.C. No Japão, o Jiu-jitsu ganha projeção e notoriedade, tendo status de "luta do samurai" e sendo considerada a mãe de todas as lutas, derivando, a partir dele, inúmeras práticas como o Aiki-jitsu, Tai-Jitsu, o Jo-Jitsu e, mais tarde, o Judô (Rufino \& Darido, 2009).

No Brasil, o Jiu-jitsu teve seu início na cidade de Belém do Pará, com a chegada do professor Mitsuyo Maeda, conhecido como Conde Koma em 1917. Conde Koma fez uma demonstração da luta e o primeiro a assistir foi Carlos Gracie, que se interessou com a forma de combate e começou a treinar ficando habilidoso apesar do seu físico não se enquadrar para um esporte de luta (Virgílio, 2002).

De acordo com Pereira et al. (2011), o Brazilian Jiu-jitsu (BJJ), é uma modalidade esportiva de combate caracterizada por esforços intermitentes de alta intensidade, intercalados por períodos de recuperação. Embora a luta seja influenciada por diversos fatores tais como táticos, técnicos e psicológicos, tem sido observado que em esportes de combate, o fator condicionamento físico pode ser determinante para o resultado final (Ratamess, 2011).

Segundo Moreira, Andréia Gulak, Ulanowicz, Ronque, De Souza Silva (2003), de todas as capacidades físicas condicionantes, a capacidade de força é uma das mais importantes a ser desenvolvida no BJJ. Desta forma, Ratamess (2011), sugere o aprimoramento das manifestações de potência, força máxima e resistência de força para o bom desempenho nesta modalidade.

Outro fator importante dentro das modalidades de lutas é a composição corporal, Uma vez que é comum os atletas buscarem a redução da massa corporal para se enquadrarem no limite inferior de determinada categoria de peso (Andreato et al., 2012).

Desta forma, de acordo com Avelar et al. (2008), a determinação do padrão morfológico e do desempenho físico de uma modalidade esportiva torna-se bastante importante para a classificação, seleção e preparação de atletas. Estudos de Falcão (2004) e Gehre, Coelho, Botelho Neto, Queiroz \& Campbell (2010), determinaram que as capacidade de força, velocidade, flexibilidade, resistência, coordenação e equilíbrio, quando combinadas, poderão gerar bons desempenhos à atletas do Brazilian Jiu-jitsu.

Contudo, ainda são poucos os trabalhos e pesquisas relacionados a este tema para a modalidade em questão, principalmente comparando praticantes do sexo masculino e feminino.

Portanto, o principal objetivo deste estudo foi de caracterizar o perfil antropométrico e funcional de atletas do sexo masculino e feminino praticantes da modalidade do Brazilian Jiu-jitsu (BJJ).

\section{Metodologia}

Participaram deste estudo 19 praticantes do Brazilian Jiu-jitsu, pertencentes ao projeto Tatame do Bem, do Instituto Federal de Educação, Ciência e Tecnologia do Sul de Minas Gerais (IFSULDEMINAS), Campus Muzambinho, dos quais 7 eram do sexo feminino (21,29 \pm 4,68 anos de idade; $25,84 \pm 3,90 \mathrm{~kg} /$ $\mathrm{m} 2$ de IMC; $22,03 \pm 8,20 \%$ de gordura corporal) e 12 do sexo masculino (22,03 \pm 6,04 anos de idade; $23,98 \pm 4,08 \mathrm{~kg} / \mathrm{m} 2$ de IMC; $14,53 \pm 8,66 \%$ de gordura corporal). Todos os voluntários, no momento da seleção da amostra apresentavam pelo menos um ano de treinamento sistematizado na modalidade, com, pelo menos, quatro horas de prática, divididas em duas sessões semanais. Com relação a graduação dos voluntários, estes apresentavam graduações que iam desde a faixa branca ( $1^{\text {a }}$ faixa) até a faixa azul ( $3^{\circ}$ faixa). 
Previamente ao estudo, todos os voluntários foram informados sobre os objetivos, benefícios e riscos da presente investigação podendo, através de um termo de consentimento livre e esclarecido, consentir ou não sua participação no mesmo. O presente trabalho atendeu as normas para a realização de estudos em seres humanos, de acordo com a resolução 196/96 do Conselho Nacional de Saúde.

\section{Procedimentos}

Todos os procedimentos foram realizados nos laboratórios do Centro de Ciências Aplicadas à Educação e Saúde (CeCAES) do IFSULDEMINAS, Campus Muzambinho.

Nos mesmos dias e horários que ocorriam os treinamentos dos atletas, estes compareceram aos laboratórios onde, inicialmente foram submetidos à avaliação antropométrica, seguida das avaliações funcionais: flexibilidade, potência de membros inferiores e força de preensão manual, necessariamente nessa ordem e respeitando o tempo de descanso de acordo com o protocolo de cada teste.

À todos os voluntários foi sugerido que comparecessem aos locais de avaliação com roupas apropriadas para a prática de atividade física, que não executassem qualquer tipo de exercício extenuante nas 24 horas que antecedessem as avaliações e que tivessem realizado sua última refeição à pelo menos duas horas antes das avaliações.

\section{Determinação da Flexibilidade}

A flexibilidade foi avaliada pelo teste de sentar e alcançar utilizando o banco de Wells (Terra Azul Physical). Ao avaliado foi pedido que sentasse no chão, com as pernas estendidas e com a sola dos pés encostadas no banco. Ao sinal do avaliador o voluntário deveria estender os braços unindo as mãos a frente do corpo, flexionar o tronco e deslizar as mãos sobre a haste graduada do banco o máximo que conseguisse. Este procedimento foi realizado em três tentativas, onde o resultado final foi a melhor medida das três execuções.

\section{Avaliação antropométrica}

Esta consistiu na determinação da altura e medidas de composição corporal. A estatura foi aferida através do Estadiômetro portátil (ES T22- Coumarg) onde, o avaliado em posição ortostática e descalço, deveria ficar de costas para a haste graduada do estadiômetro. Após a realização de uma inspiração forçada a haste de medida do estadiômetro era colocada sobre o ápice da cabeça determinando a altura do voluntário (Alt).

A composição corporal foi determinada através de uma balança de bioimpedância tetrapolar (InBory720). Aos voluntários foi solicitado que respeitassem alguns critérios para a maior confiabilidade dos resultados: a) ter realizado a última refeição a pelo menos 2 horas antes; b) ter evacuado anteriormente a análise; c) Não realizar exercícios físicos nas 24 horas que antecederam a avaliação; d) permanecer pelo menos 5 minutos na posição ortostática; e) não tomar banho ou sauna momentos antes da avaliação (Eickemberg, Oliveira, Roriz \& Sampaio, 2011).

Para avaliar a potência de membros inferiores foi utilizado um tapete de contato (Jump System Pro CEFISE) e a técnica de salto Squat Jump. Os avaliados foram orientados a posicionarem-se no tapete de contato e ficarem em uma posição de $90^{\circ}$ graus de flexão de joelhos conhecido como meio agachado e as mãos apoiadas na cintura. Ao sinal do avaliador, os voluntários deveriam realizar um salto no sentido vertical o 
mais alto possível sem a ajuda dos braços. Cada voluntário realizou três tentativas separadas por 30 segundos de descanso. Das três tentativas, o melhor resultado foi considerado como medida de potência de membros inferiores.

\section{Força de Preensão Manual}

Para o teste de força de preensão manual foi utilizada o dinamômetro manual da marca (Dinamômetro Manual 50Kgf Crown). $\mathrm{Na}$ avaliação o voluntário deveria permanecer em pé, com afastamento lateral das pernas, os braços ao longo do corpo, o punho e antebraço em posição de pronação, segurando confortavelmente o dinamômetro com a escala de medida voltada para o avaliador, que ao sinalizar, o avaliado deveria, através da flexão dos dedos das mãos, exercer o máximo de força possível por um período de 5 segundos. De forma a evitar qualquer movimento compensatório, o avaliado foi orientado a manter os demais membros corporais imóveis durante o período de avaliação. Foram realizadas três tentativas separadas por um período de descanso de um minuto entre elas, onde o melhor resultado das três tentativas foi adotado como medida para a presente investigação.

Todos os dados estão expressos em média e desvio padrão da média. Estes, foram submetidos à uma análise de normalidade através do teste Shapiro Wilk. Diante da averiguação de normalidade de todas as variáveis do presente estudo, para a determinação das possíveis diferenças entre as variáveis estudadas entre os gêneros (masculino e feminino), foi aplicado o teste $t$ de Student para amostras independentes. Todas as análises foram feitas através do pacote estatístico BioEstat versão 5.0, sendo adotado um nível de significância de $\mathrm{p} \leq 0,05$.

A amostra da presente investigação foi dividida em dois grupos de acordo com o gênero (masculino e feminino). $\mathrm{Na}$ Tabela 1 , são apresentados os dados em média e desvio padrão da média desses grupos. Nesta, pode-se verificar que as variáveis, altura, preensão manual dos braços direito e esquerdo, altura, potência absoluta e potencia relativa do salto vertical foram significantemente diferentes entre os grupos. As demais variáveis, idade, peso corporal, IMC, porcentagem de gordura e flexibilidade, não apresentaram diferenças estatísticas.

TABELA 1:

Valores em média e desvio padrão da média das características da amostra. Comparação entre gênero

\begin{tabular}{|l|l|l|}
\hline & Masculino $(\mathrm{n}=12)$ & Feminino $(\mathrm{n}=7)$ \\
\hline Idade (anos) & $22,91 \pm 6,40$ & $21,28 \pm 4,68$ \\
\hline Peso (kg) & $71,59 \pm 13,31$ & $67,17 \pm 15,37$ \\
\hline Altura (cm) & $172,66 \pm 4,20$ & $160,42 \pm 7,59^{*}$ \\
\hline IMC (kg/m2) & $23,97 \pm 4,08$ & $25,84 \pm 3,90$ \\
\hline \% Gordura (\%) & $14,53 \pm 8,65$ & $22,02 \pm 8,20$ \\
\hline PMdir. (Newton) & $47,35 \pm 8,77$ & $30,28 \pm 6,65^{*}$ \\
\hline PMesq. (Newton) & $40,75 \pm 8,45$ & $25,57 \pm 5,94^{*}$ \\
\hline Alt.SV (cm) & $33,34 \pm 4,98$ & $23,33 \pm 4,30^{*}$ \\
\hline PotAbs.SV (Watts) & $3226,79 \pm 517,63$ & $2526,01 \pm 701,86^{*}$ \\
\hline PotRel.SV (Watts.kg-1) & $45,29 \pm 4,36$ & $35,38 \pm 4,68^{*}$ \\
\hline Flex. (cm) & $26,75 \pm 7,15$ & $26,71 \pm 7,13$ \\
\hline
\end{tabular}

* diferença significante com relação ao gênero masculino - p $\leq 0,05$; IMC = índice de massa corporal; PMdir. = preensão manual para $o$ braço direito; PMesq. = preensão manual para o braço esquerdo; Alt.SV = altura do salto vertical; PotAbs.SV = potência absoluta do salto vertical; PotRel.SV = potência relativa do salto vertical; Flex. = teste de flexibilidade. 
O presente estudo teve como objetivo caracterizar o perfil antropométrico e desempenho físico de atletas praticantes da modalidade do Brazilian Jiu-jitsu (BJJ) pertencentes ao projeto de extensão "Tatame do Bem” do Instituto Federal de Educação, Ciência e Tecnologia do Sul de Minas Gerais (IFSULDEMINAS), Campus Muzambinho. Até mesmo para uma melhor caracterização dos praticantes dessa modalidade, os atletas foram divididos de acordo com o sexo (masculino e feminino). Onde, todas as variáveis estudadas foram comparadas entre homens e mulheres. Destas, principalmente àquelas relacionadas a capacidade de força muscular apresentaram diferenças significantes.

Com relação a capacidade de força muscular, Monteiro (1997) relata que, quando expressa em valores absolutos, os homens tendem a apresentar maiores níveis de força que as mulheres. As mulheres, de acordo com Fleck \& Kraemer (2006), apresentam níveis de força, cerca de dois terços dos valores apresentados pelos homens. Contudo, quando a força é expressa de forma relativa ao peso corporal, a diferença entre os sexos tende a diminuir drasticamente (Fleck \& Simão, 2008).

De acordo com Fleck \& Simão (2008), da mesma forma que a capacidade de força muscular, a potência muscular, quando expressa em valores absolutos é superior em homens. De acordo com os autores, o desempenho no salto vertical tende a ser mais baixo em mulheres. Porém, quando expressa de forma relativa, semelhante a capacidade de força, a lacuna entre os sexos tende a se estreitar. Todos esses aspectos poderiam explicar as diferenças encontradas entre homens e mulheres com relação as variáveis destinadas a capacidade de força e potência muscular para a presente investigação.

Com relação a força de preensão manual, Andreato et al. (2012), verificaram uma queda de 11 e 16\% para os braços direito e esquerdo, respectivamente, após a realização de lutas da modalidade de Jiu-jitsu. Contudo, os autores salientam que a força de preensão manual não aparenta ser uma característica importante para a modalidade, haja vista, os atletas de Jiu-jitsu, no geral apresentarem níveis baixos para esta variável.

Gehre, Coelho, Botelho Neto, Queiroz \& Campbell (2010), analisando o nível de aptidão física de praticantes de Jiu-jitsu de diferentes níveis e comparando-os com um grupo controle não praticante da modalidade, verificaram que o teste de potência muscular para membros superiores (arremesso do Medicine Ball) era muito mais discriminatório para o nível de força entre os diferentes grupos do que o teste de potência muscular para membros inferiores. De acordo com os autores, este resultado expressa a possível ênfase da força de membros superiores para a modalidade quando comparada a força de membros inferiores.

Silva, Ide, Simim, Maracolo \& Mota (2014) salientam que a força estática para membros superiores perece ser mais importante para atletas de Jiu-jitsu quando comparada a força dinâmica para membros inferiores. No entanto, algo que pode explicar a manutenção das variáveis de força estática diante de uma sessão de treinamento, seria a alta variabilidade da presente amostra para esta variável (coeficiente de variação superior a 20\%), diminuindo o poder estatístico.

Com relação a composição corporal, Andreato et al. (2012), analisando atletas altamente treinados em $\mathrm{BJJ}$, verificaram que o percentual de gordura destes atletas foi semelhante aqueles apresentados por atletas de modalidades de combate e abaixo da média populacional para a mesma faixa etária. Andreato et al. (2012) encontraram valores de percentual de gordura que variavam entre $7 \%$ e $15 \%$, indo de encontro aos resultados da presente investigação, onde os atletas do sexo masculino apresentaram valores médios de $14,3 \%$. Além disso, Franchini, Nunes, Moraes \& Del Vecchio (2007), analisando esta variável em atletas universitários de Judô, encontraram valores semelhantes ao da presente amostra, por volta de $14 \%$. Vale ressaltar que todos os atletas voluntários do presente estudo, eram atletas universitários. 
Roas, Souza \& Brito (2009) investigaram a prevalência de estratégia de rápida redução de massa corporal em lutadores de Jiu-jitsu, no qual as características antropométricas das mulheres lutadoras estavam dentro do padrão de normalidade, por volta de $19 \%$ de gordura e IMC por volta de $22 \%$, resultados prximos ao da presente investigação, com percentual de gordura por volta de $22 \%$ e IMC de por volta de $25 \%$. Devese considerar ao se comparar o percentual de gordura entre os sexos que, em média, os homens apresentam percentuais de gordura mais baixos que as mulheres, por volta de $15 \%$ e $25 \%$, respectivamente (McArdle, Katch \& Katch, 1991). Desta forma, um dos principais motivos que possivelmente determinou a igualdade estatística entre os gêneros para esta variável na presente investigação, foi a grande variabilidade das amostras recrutadas, ou seja, devido a falta de atletas que se encaixassem adequadamente nos critérios de inclusão da presente investigação, foi desconsiderado a questão categoria dos avaliados, o que pode ter contaminado a amostra com relação a composição corporal e em especial, o percentual de gordura.

Dos testes motores, o de flexibilidade foi o único que não apresentou diferenças significantes entre os gêneros, corroborando com os achados de Araujo, Oliveira, Paz \& Santos (2002). Existem fatores que influenciam nos graus de flexibilidade, estes podem ser divididos em endógenos e exógenos, dos quais destacam-se a temperatura ambiente, hora do dia, idade, sexo, somatotipo, individualidade biológica e condição física (Dantas, 1999). As mulheres por possuírem tecidos menos densos são normalmente mais flexíveis que os homens (Dantas, 1999), contudo, deve-se considerar que não só o sexo é um fator determinante no nível de flexibilidade, o que poderia explicar a igualdade dessa variável na presente investigação.

Segundo Farintti (2000), a flexibilidade é um componente importante da aptidão física, se define como uma maior amplitude articular para execução de qualquer gesto. Esta capacidade é bastante importante para a prática de uma arte marcial como o Jiu-jitsu, tanto para a defesa quanto para o ataque (Andreato, 2014). Na pratica do Jiu-jitsu as articulações toraco-lombar e de quadril são constantemente solicitadas, principalmente no trabalho de "solo" e especialmente no trabalho de guarda, tornando-se dessa forma, uma das capacidades físicas a ser considerada no processo de avaliação e treinamento para a modalidade (Souza, Silva \& Camões, 2005).

Souza, Silva \& Camões (2005), tiveram como objetivo, avaliar a flexibilidade toraco-lombar e de quadril em atletas de Jiu-jitsu, os resultados revelaram uma forte associação entre o tempo de prática da modalidade e a flexibilidade dessa região e que esta capacidade, é aumentada sob a influência do treinamento. Castro, Venâncio, Doimo \& Locatele (2008) e Del Vecchio, Stefania, Hirata \& Chacon- Mikahil. (2007) encontraram valores de flexibilidade no teste de sentar e alcançar em atletas de judô e Jiu-jitsu do sexo masculino, respectivamente, cerca de $10 \mathrm{~cm}$ acima daqueles encontrados na presente investigação para o mesmo sexo. Por outro lado, Amorin, Albertini, Felix, Magalhães \& Soares (2015) verificaram níveis inferiores de flexibilidade em atletas de judô do sexo masculino quando comparados a presente amostra. Contudo, deve-se ressaltar que o tempo de prática desses atletas era inferior ao tempo de prática da presente amostra, corroborando a afirmação de Souza, Silva \& Camões (2005).

Desta forma, quando se faz um paralelo entre os resultados apresentados pela amostra do presente estudo com a amostra de outros estudos, verifica-se um possível menor nível de treinamento, acarretando para aquelas capacidades menos expoentes durante a luta a igualdade entre os sexos, algo presente na capacidade de flexibilidade.

Pode-se concluir com o presente estudo que quando comparadas as características físicas de praticantes de Jiu-jitsu de ambos os sexos, os homens apresentam maiores níveis de força em relação as mulheres e que estas diferenças, para a população estudada não se estendeu às características antropométricas e à capacidade de flexibilidade 


\section{REFERÊNCIAS}

Andreato, L. V; Franchini, E; Moraes, S. M. F; Esteves, J. V. D. C; Pastório, J.J ; Gomes, T. L. M; Vieira, J. L. L. (2012). Perfil morfológico de atletas de elite de Brazilian Jiu-jitsu. Revista Brasileira de Medicina do Esporte. 18(1), 46-50.

Andreato, L. V. (2014). Respostas fisiológicas e análise técnico tática em atletas de Brazilian jiu-jitsu submetidos à luta fragmentada e simulação de competição. (Dissertação de mestrado) Pós Graduação em Estudos do Esporte Universidade de São Paulo, Escola de Educação Física e Esportes (EEFE-USP). São Paulo: USP. $116 f$.

Amorin, D. A. S.; Albertini, L. C. B.; Felix, S. H.; Magalhães, T. T.; Soares, W. D. (2015). Flexibilidade em praticantes e não praticantes de judô. Revista Ensino \& Pesquisa. 13(1), 136-147.

Araujo, S. S.; Oliveira, H.; Paz, A. A.; Santos, C. A. S. (2002). Avaliação da flexibilidade de adolescentes através do teste de sentar e alcançar. Revista Digital Vida \& Saúde, 1(1), 1-5.

Avelar, A.; Santos, K. M.; Cyrino, E. S.; Carvalho, F. E.; Dias, R. M. R.; Altimari, L. R.; Gobbo, L. A. (2008). Perfil antropométrico e de desempenho motor de atletas paranaenses de futsal de elite. Revista Brasileira de Cineantropometria e Desempenho Humano, 10(1), 76-80.

Castro, E. A.; Venâncio, R. A. F.; Doimo, L. A.; Locatele, J. (2008). Comparação entre dois métodos de treinamento para aprimoramento da flexibilidade em judocas da associação atlética academia- LUVE/UFV. Coleção Pesquisa em Educação Física, 7(2), 195-202.

Dantas, E. H. M. (4a Ed.). (1999). Flexibilidade, Alongamento e Flexionamento. Rio de Janeiro: Shape.

Del Vecchio, F. B.; Stefania, B.; Hirata, S. M.; Chacon-Mikahi, M. P. T. (2007). Análise morfo-funcional de praticantes de brazilian jiu-jitsu e estudo da temporalidade e da quantificação das ações motoras na modalidade. Movimento e Percepção, 7(10), 263-281.

Eickemberg, M.; Oliveira, C.C.; Roriz, A.K.C.; Sampaio, L.R. (2011). Bioimpedância elétrica e sua aplicação em avaliação nutricional. Revista de Nutrição, 24(6), 883-93.

Falcão, A. P. S. T. (2004). Modelagem para deteç̧ão de talentos desportista em judocas. (Dissertação de Mestrado), Programa de Pós Graduação em Biometria - Universidade Federal Rural de Pernambuco. Recife: UFRPE. $48 f$.

Farinatti, P. T. V. (2000). Flexibilidade e esporte: uma revisão de literatura. Revista Paulista de Educação Física, 14(1), 85-96.

Farinatti, P. T. V.; Monteiro, W. D. (2a Ed.). (1992). Fisiologia e Avaliação Funcional. Rio de Janeiro: Sprint.

Fleck, S ; Simão, R. (2008). Força princípios metodológicos para o treinamento. São Paulo: Phorte.

Fleck, S. J.; Kraemer, W. J. (3 ${ }^{\mathrm{a}} \mathrm{Ed}$.). (2006). Fundamentos do treinamento de força muscular. Porto Alegre: Artmed.

Franchini, E.; Nunes, A. V.; Moraes, J. M.; Del Vecchio, F. B. (2007). Physical fitness and anthropometrical profile of the Brazilian male judo team. Journal of Physiological Anthropology, 26(2), 59-67.

Gehre, J; Coelho J; Botelho Neto W; Queiroz J; Campbell C. (2010). Aptidão física de alunos do ensino médio praticantes e não praticantes de Jiu-jitsu. Revista Brasileira de Ciência e Movimento, 15(2), 76-83.

McArdle, W; Katch, F. I; Katch, V. L. (1991). Fisiologia do Exercício: energia, nutrição e desempenho humano. Rio de Janeiro: Guanabara Koogan.

Monteiro, W. D. (1997). Força muscular: uma abordagem fisiológica em função do sexo, idade e treinamento. Revista Brasileira de Atividade Física e Saúde, 2(2), 50-66.

Moreira, S. R.; Andréia Gulak. A.; Ulanowicz, J. S.; Ronque, E. R. V.; De Souza Silva, K. E. (2003). Correlação de variáveis antropométricas de membros superiores com a força de preensão manual em praticantes de Jiu-Jitsu. Motriz, 9(1), S147.

Pereira, R. F.; Lopes, C. R.; Dechechi, C. J.; Silva, B. V. C.; Ide, B. N.; Navarro, A. C. (2011). Cinética de remoção de lactato em atletas de Brazilian jiu-jítsu. Revista Brasileira de Prescrição e Fisiologia do Exercício, 5(25), 34-44.

Ratamess, N. A. (2011). Strength and Conditioning for Grappling Sports. Strength and Conditioning Journal, 33(6), $18-24$.

Roas, A. F. C. M.; Souza, E. R.; Brito, C. J. (Setembro, 2009). Prevalência de estratégia de rápida redução de massa corporal em lutadores de Jiu-jitsu. Trabalho publicado nos Anais do XVI Congresso Brasileiro de Ciências do 
Esporte e III Congresso Internacional de Ciências do Esporte, Salvador, Brasil.

Rufino, L. G. B.; Darido, S. C. (2009). Considerações iniciais sobre o jiu jitsu brasileiro e suas implicações para a prática pedagógica. Trabalho publicano nos Anais do Congresso Paulistano de Educação Física Escolar, Caraguatatuba, Brasil.

Silva, B. V. C.; Ide, B. N.; Simim, M. A. M.; Marocolo, M.; Mota, G. R. (2014). Neuromuscular Responses to Simulated Brazilian Jiu-Jitsu Fights. Journal of Human Kinetics, 44(1), 249-257.

Souza, I.; Silva, V.S.; Camões, J. C. (2005). Flexibilidade toraco-lombar e de quadril em atletas de jiu-jitsu. EFDeportes: Revista Digital, (82).

Virgílio, S. (2002). Personagens e Histórias do Judô Brasileiro. Campinas: Átomo. 